ganz unaufgeklärt sind - ob z. B. der nv. depressor die Erregung der gefässverengenden Nerven aufhebt oder reflectorische Reizung der Gefässerweiterer veranlasst, ist meines Wissens durchans unentschieden, - so ist wohl klar, dass mit der Bezeichnung "Hemmungswirkung" nur der Hinweis auf ein äusseres Verhalten gegeben ist, dessen nähere Bestimmung und innere Charakteristik in jedem besonderen Falle näher untersucht werden muss.

Deshalb wäre es auch durchaus falsch, aus dem Nachweise, dass in dem nv. vagus die Hemmungs- und Erregungsfunction an verschiedene Classen von Nervenfasern gebunden ist, den Schluss zu ziehen, dass dieses Verhalten ein allgemeines sei. Niemand wird sich wohl entschliessen, in den Stämmen der Hautnerven reflexerzeugende und reflexverhindernde Fasern nebeneinander anzunehmen, obwohl von der Haut aus Bewegungen reflektorisch sowohl hervorgerufen als unterdrückt werden können. Die fernere Untersuchung der sogenannten Hemmungsvorgänge nach den bezeichneten Richtungen hin bildet eine der dankbarsten und interessantesten Aufgaben für die weitere Forschung.

\title{
Zur Frage nach der Rindenlocalisation beim Menschen.
}

Von

Prof. Sigm. Exner,

Assistenten am physiologischen Institute in Wien.

In der am 5. Jänner 1882 erschienenen Nummer der "Nature“ findet sich eine Besprechung meines jüngst erschienenen Buches „Untersuchungen über die Localisation der Functionen in der Grosshirnrinde des Menschen", Wien 1881. Dieselbe ruihrt von Ferrier her, und beurtheilt dieses Buch im Gegensatz zu mancher anderen Kritik nicht günstig. Es beruht dieses abfällige Urtheil zum grossen Theil auf Bedenken, von denen ich mir erwartet hatte, dass sie von manchen, die das Buch nicht genauer durchsähen, getheilt 
würden. Obwohl ich in dieser Beziehung bei der Abfassung desselben schon vorgebeugt zu haben glaube, ergreife ich doch die Gelegenheit, welche mir Ferrier durch seine Kritik bietet, auf gewisse Einwände näher einzugehen, im Glauben, hierdurch das Urtheil auch Anderer rectificiren zu können.

Ich bin mit Ferrier vollkommen einverstanden, wenn er im Eingang zu seinem Artikel auf die grossen Schwierigkeiten hinweist, mit denen es verbunden ist, aus Krankheitsfällen und Sectionsbefunden richtige Schliusse auf die Localisation der Hirnfunctionen zu ziehen. Ueber diese Localisation beim Menschen können wir aber vorläufig nur auf diesem Wege etwas erfahren, und die Aufgabe, die das genannte Buch zu lösen suchte, bestand eben darin, diejenigen Thatsachen, und seien es anch nur wenige, welche sich trotz dieser Schwierigkeit mit Bestimmtheit feststellen lassen, zu ermitteln. Wenn aber Ferrier pag. 215 sagt: „Apart altogether from Prof. Exner's deductions, one thing clearly brought out by the facts is the comparative impotence of the fortuitons experiments of disease as regards the determination with any degree of accuracy of the exact position and limits of any centre whatever" 1 ), so muss ich fragen: warum? Wenn ich alle untadelhaft beschriebenen Krankengeschichten und Sectionsbefunde der medicinischen Literatur, welche bestimmten für den vorliegenden Zweck als nothwendig erkannten Regeln entsprechen, ohne mich in der Auswahl derselben durch den Wunsch, irgend etwas beweisen oder widerlegen zu wollen, beeinflussen zu lassen, in eine Sammlung zusammenstelle, und dann alle jene Läsionen, welche im Leben keine Motilitätsstörung, z. B. der rechten oberen Extremität, im Gefolge hatten, auf einem Tableau der Hirnoberfläche auftrage, so finde ich die ganze Oberfläche von solchen Zeichnungen bedeckt, mit Ausnahme einer ganz bestimmten Region der linken Hemisphäre. Ich finde an der anderen Hemisphäre auf demselben Weg eine analog gelegene Region für die linke obere Extremität. Wenn ich weiter finde, dass einzelne Stellen dieser Region in den Fällen

1) „Sehen wir vorläufig ab von Prof. Exner's Folgerungen, so ergiebt sich mit voller Klarheit ein Umstand aus den vorgebrachten Thatsachen: die dem Spiele des Zufalles anheimgestellten Experimente der Natur, die uns in Form von Krankengeschichten vorliegen, sind verhältnissmässig unfähig, uns die Lage und Begrenzung irgend eines Centrums in einigermaassen verlässlicher Weise zu lehren." 
meiner Sammlung bis zu 20 mal und öfter Sitz einer Läsion waren und dann immer Motilitätsstörungen der betreffenden Extremitäten vorhanden waren, beweist das nichts für die Bedeutung dieser Rindenstellen? So sind meine absoluten Rindenfelder aufgefunden, und was die Genauigkeit ihrer Grenzen anbelangt, so ist nicht zu vergessen, dass man über einen gewissen, ziemlich geringen Grad schon wegen der anatomischen Variationen der Hirnoberfäche weder beim Menschen noch beim Thiere gelangen kann. Dieser Grad dürte aber durch meine Methode sehr wohl erreichbar, vielleicht schon erreicht sein. Jedenfalls ist sie mehr dazu geeignet, als Experimente an Thieren, und die Uebertragung: der vorgefundenen Resultate auf das Gehirn des Menschen.

Ferrier sagt weiter: „in his selection of instances he has excluded, without assigning valid reasons, many cases of the utmost importance"). Nun kann ich freilich nicht behaupten, dass mir nicht ein einziger wichtiger Krankenfall der Litteratur entgangen ist, aber "ausgeschlossen", d. h. in meinem Katalog als "unbrb." bezeichnet, habe ich keinen, welcher nicht nach den pag. 7 meines Buches aufgestellten Angaben verworfen werden musste. Ferrier luätte demnach diese Regeln oder die Methode iuberhaupt nach Regeln die Fälle auszuwählen, angreifen müssen, dann hätte ich auf diesen Vorwurf antworten können; auf den obigen allgemeinen Satz kann ich nur erwidern, dass er meines Erachtens unrichtig ist.

Einen grossen Fehler meiner Untersuchungen findet Ferrier weiter darin, dass ich Fälle von Hirntumoren mit in meine Betrachtungen gezogen habe, "welche nach allgemeiner Uebereinkunft der Pathologen auszuschliessen" sind. Da Ferrier kurz vorher hervorhob, dass ich Physiolog sei, so könnte man glauben, ich hätte diesen Fehler in Unkenntniss der genannten Auffassung der Pathologen begangen. Pag. 16 hob ich aber ausdrücklich hervor, dass und warum ich "die Ansicht mancher Pathologen" über die Wirknng eines Hirntumors nicht theilen kann, und Ferrier hätte also, anstatt die alte Ansicht mancher Pathologen zu wiederholen, zeigen sollen, dass meine neue Ansicht und die Gründe dafïr unrichtig sind.

1) "Aus seiner Sammlung von Krankheitsfällen hat er ohne Angabe eines hinreichenden Grundes viele Fälle ausgesehlossen, die von der grössten Wichtigkeit sind." 
Besonders abgeneigt zeigt sich Ferrier meinen „relativen Rindenfeldern" und dem Umstande, dass die Rindenfelder verschiedener Körperorgane ineinanderfliessen, theilweise sogar eines in dem andern eingeschlossen ist. Er meint: „if Prof. Exners localisation of relatives centres had any foundation in fact, it would be nothing short of a reductio ad absurdum of the whole doctrine of localisation .... A consistent advocate of localisation might as well admit that the leg may frequently, relatively, or in some way or other be used as an organ of vision."

Was die relativen Rindenfelder anbelangt, so sind sie nicht der Inhalt einer willkürlich von mir aufgestellten Theorie, sondern sie sind der in eine klare Form gebrachte Ausdruck der Thatsachen. Es kommt eben, wie Ferrier kaum in Abrede stellen diirfte, vor, dass die Läsion ein und derselben Rindenstelle bei einem Kranken von einem gewissen Symptom begleitet ist, bei einem anderen nicht. Ich verweise in dieser Beziehung auf die, pag. 35 meines Buches, speciell hierauf gerichteten Untersuchungen. Wenn ich sage, meine relativen Rindenfelder sind der Ausdrnck für Thatsachen, so meine ich damit, dass ich iber die Art, wie sie sich an dem Zustandekommen einer willkürlichen Bewegung betheiligen, durchaus keine bestimmte Aeusserung thun will hierüber wissen wir auch bei den absoluten Rindenfeldern nichts es mag sich also jeder darüber seine eigenen Gedanken machen; ich muss es aber als ungerechtfertigt zurïckweisen, wenn jemand das Zusammenfallen einer Läsion mit dem Fehlen gewisser Functionen einmal, wo es zu seinen vorgefassten Anschauungen passt, als Beweis dafür anffasst, dass die lädirte Hirnstelle das "Centrum ${ }^{4}$ für diese Functionen ist, das anderemal aber, wo es zu seinen Anschauungen nicht passt, von einer Fernwirkung der erkrankten Stelle auf jenes hypothetische "Centrum" spricht. Eine solche Fernwirkung kann nur in zweierlei Sinn verstanden werden. 1. Entweder es werden durch die Läsion Nervenbahnen zerstört, welche in physiologischen Zusammenhang mit den Bahnen des

1) "Wenn Prof. Exner's Localisation der relativen Rindenfelder auf Thatsachen beruhte, so wäre dadurch die ganze Localisationslehre geradezu ad absurdum geführt" .... „Ein consequenter Anwalt der Localisationslehre fände das gerade so, wie die Behauptung, dass das Bein häufig, oder unter gewissen Umständen, oder auf die eine oder die andere Weise als Organ des Gesichtssinnes gebraucht werden könne."

E. Pflüger, Archiv f. Physiologie. Bd, XXVII. 
"Centrums" stehen, und es wird die Function derselben eben dadureh gestört, dass er von seinen normalen Verbindungen abgetrennt wird: dann gehört die lädirte Stelle eben noch mit zum "Centrum", und steht mit diesem nur in loserem Zusammenhange, als die Theile des "Centrums" untereinander; es wäre dieses eine Deutung für meine relativen Rindenfelder. 2. Oder es wird voransgesetzt, dass die erkrankte Stelle durch eine für das Auge vollkommen normal erscheinende Strecke der Hirnrinde hindurch irgend einen räthselhaften Einfluss auf die Functionen des "Centrums" ausübt. Hält man an dieser Voraussetzung fest, dann ist fast nur durch meine "Methoden der negativen Fälle und der procentischen Berechnung" überbaupt etwas über die Localisation zu ermitteln, die gangbare "Methode der positiven Fälle" ist dann ganz verwerflich. Speciell meine Aufstellungen der Rindenfelder auf Grund der "Methode der negativen Fälle" sind unabhängig von jeder Fernwirkung, es müsste denn sein, dass man bei jeder Läsion eine solche Fernwirkung der letzteren Art voraussetzen wollte. Ja, diese Methode liefert noch richtige Resultate, es mögen ausser der in Frage kommenden Hirnläsion noch irgend beliebige andere Erkrankungen im Centralnervensystem vorhanden sein.

Ich hebe dieses hier hervor, um dem Missverständniss entgegenzutreten, dass die Resultate meiner Untersuchungen auf Kosten ihrer Richtigkeit durch die sogenannten Fernwirkungen wesentlich beeinflusst worden wären. Es kann das für die absoluten Rindenfelder nicht der Fall sein, und was die relativen anbelangt, so zweifle ich nicht an der Existenz einer Fernwirkung der ersten Art, finde vielmehr in ihr einen Anhaltspunkt fur die Deutung dieser Rindenfelder, und glaube, dass sehr oft wo von Pathologen ein Symptom durch Fernwirkung erklärt werde, diese Art derselben vorlag. Ich zweifle auch nicht an der Existenz der, so viel ich weiss, bisher allein beachteten zweiten Art der Fernwirkung, möchte aber darauf aufmerksam machen, dass wenn man meine relativen Rindenfelder anf Grund dieser erklären will, man in eigenthümliche Schwierigkeiten geräth. Man müsste nämlich eine derartige durch normal erscheinende Rindenantheile ausgeübte Fernwirkung auf viele Centimeter, ja fast um die Hälfte einer Hemisphäre herum annehmen, und zwar von unbedeutenden keine nennenswerthen Circulationsstörungen bedingenden Läsionen. Von Erkrankungen im Gebiete des Rückenmarkes und des Gehirn- 
stammes wissen wir, dass eine Fernwirkung auf so grosse Distanzen kaum je vorkommt. Wie oft liegt da ein Heerd nahe an einem Nervenkern der ganz normal fungirt. Aber selbst wenn jemand die Annahme von einer so weit reichenden Fernwirkung machen wollte, so würde ihn das seinem Ziele die relativen Rindenfelder los zu werden, nicht näher führen. Diese bleiben nämlich nach wie vor für alle jene Maskelgruppen bestehen, welche gar keine absoluten Rindenfelder haben. Oder zweifelt Ferrier daran, dass man eine Hemisphäre mit Zeicbnungen von Läsionen bedecken kann, welche alle ohne Lähmung der gegenüberliegenden Augenmuskeln oder Zungenmuskeln bestanden haben? In meiner Sammlung finden sich Fälle genug um das zeigen zu können. Bei Anwendung der "Methode der negativen Fälle" könnte die hypothetische Fernwirkung nur dazu beitragen ein solches "Centrum" grösser erscheinen zu lassen, als es wirklich ist und doch findet man nach dieser Methode keines.

Handelt es sich also um eine physiologische Deutung meiner relativen Rindenfelder, so bin ich geneigt eine Fernwirkung der zweiten Art auf die uns aus dem Gehirnstamm bekannt gewordenen Distanzen anzunehmen, da aber wo diese Distanzen um ein Vielfaches übertroffen würden, die Deutung in einer Fernwirkung der ersten Art zu suchen, in soferne iuberhaupt eine Fernwirkung als Erklärung nothwendig ist.

Letzteres muss ich deshalb hervorheben, weil ich in neuerer Zeit durch Untersuchungen an Thieren, auf welche Ferrier gewiss mit Recht so grosses Gewicht legt, festgestellt habe, dass auch bei diesen ein Analogon der relativen Rindenfelder des Menschen vorhanden ist, und dass hier von dem relativen Rindenfelde ans, Stabkranzfasern in die weisse Substanz der Hemisphäre eindringen um zu der betreffenden Muskelgruppe zu gelangen. (Wiener Akad. der Wiss. 14. Juli 1881.) Es zeigte sich nämlich bei Reizversuchen an Kaninchen, dass gewisse Bewegungen einer Pfote sich von einer grossen Fläche der Grosshirnrinde des Kaninehens auslösen lassen, dass aber in der Mitte dieser Fläche die schwächsten Ströme hiezu ausreichten, und dass um so stärkere angewendet werden mussten je weiter man sich von der Mitte entfernte. Trotzdem wurden auch die in den letzteren Fällen ausgelösten Bewegungen durch Fasern vermittelt, welche von der gereizten Stelle direct in die weisse Substanz eindringen. Ich denke 
dass hierdurch die Thatsache der relativen Rindenfelder endguiltig entschieden, sowie auch die physiologisehe Deutung derselben soweit angebahnt ist, dass das in vagem sinne ausgesprochene Wort "Fernwirkung" dieser Erkenntniss keinen weiteren Eintrag than wird.

Was nun den Umstand anlanģt, dass nach den Resultaten meiner Untersuchungen Rindenfelder verschiedener Functionen theilweise ineinanderfliessen, und dass ein Rindenfeld im anderen liegen kann, so muss ich auch hievon sagen, es ist das der Ausdruck der Thatsachen. Dieselbe Logik, die Prof. Ferrier damals anwendete, als er sagte: nach der Läsion der Hirnstelle a tritt Lähmung des Armes ein, also ist a das „Centrum" des Armes, wende ich jetzt an, wenn ich sage nach Läsion in $b$ tritt Lähmung von Arm und Fuss ein, also ist $b$ das "Centrum" von Arm und Fuss. Uebrigens ist dieser Schluss zuerst nicht von mir, sondern von französischen Autoren gezogen worden, wenigstens in so weit er die beiden Extremitäten betrifft. (Vgl. Lépine, Localis. dans les mal. cérébr. Thèse Paris 1875 und Charcot et Pitres. Revue mens. 1877.)

Ist nun meine Anschauung wirklich so widersinnig wie der von Ferrier in Parallele gestellte Satz: das Bein könne gelegentlich als Organ des Gesichtsinnes gebraucht werden. Ich glaube nicht. Es giebt keine Thatsache, die dafür spräche dass ein als "Centrum" in der Gehirnrinde bezeichneter Rindenantheil keine andere Function habe als die, welche Veranlassung war ihm den Namen „Centrum" zu geben; es giebt auch keine Thatsache, welche dafür spräche dass diese „Centren“, scharf umschrieben wären, dass bis an eine, wie anf einer Landkarte verzeichneten Grenze das Organ des Beines reiche, und dass jenseits dieser Linie ein anderes Organ beginne. Die Anatomie und Histologie der Rinde welehe lehrt, dass von jeder Stelle derselben Fasern in die benachbarten Rindenantheile in ungeheurer Quantität (vergl. Sigm. Exner, Wiener Akademie d. Wissensch. 3. Febr. 1881) übertreten, spricht entschieden gegen eine solche Anschaunng, und es ist das Resultat einer vorgefassten Meinung, wenn man jeden Krankheitsfall als unrein betrachtet und verwirft, in welchem, trotzdem eine Läsion sebr klein ist, Motilitätsstörungen in beiden Extremitäten vorhanden waren. Ich halte die einem solchen Vorgehen zu Grunde gelegte Anschaung, nach welcher eine als motorisches "Centrum“ 
erkannte Rindenstelle nur zu der ihr angehörigen Muskelgruppe, und sonst zu nichts anderem eine Beziehung haben kann, für eine die unbefangene Forschung behindernde Hypothese, und wundere mich dass Ferrier sich von dieser noch nicht frei gemacht hat, da es doch gerade diese Auffassung gewesen ist, die ihn seiner Zeit verleitet hat, das "Centrum" für den Tastsinn in das Subiculum cornu Ammonis, das für das Gemeingefühl in den Hinterhauptlappen zu verlegen u. d. m., woran er wohl jetzt selbst nicht mehr glauben dürfte.

Uebrigens ist es ïberflüssig sich weiter auf theoretische Anschaungen zu berufen, da ich in der oben angeführten experimentellen Untersuchung nachgewiesen habe, dass man von dem grössten Theil der von oben sichtbaren Hirnrinde des Kaninchens durch elektrische Reizung Bewegung in beiden Vorderpfoten hervorrufen kann, und dass diese Bewegungen durch Fasern ausgelöst werden, welche von der gereizten Stelle in die weisse Substanz der Hemisphäre eindringen.

Daraus folgt nun doch wohl nicht, dass das Kaninchen sein rechtes Vorderbein gelegentlich als linkes benutzen kann. Ebenso wenig folgt aus meinen Aufstellungen, dass das Bein als Gesichtsorgan gebraucht werden könne.

${ }_{n}$ Among other strange things in this book ${ }^{1}$ " findet Ferrier auch folgendes. In meiner auf "der procentischen Berechnung beruhenden Tafel XVII ist eine Stelle der medialen Fläche des Occipitallappens so gefärbt, als gehörte sie dem absoluten Rindenfeld der oberen Extremität an, d. b. als wäre ihre Verletzung in hundert Procenten der Fälle von Motilitätsstörungen begleitet. Nun ist sie aber überhanpt nur einmal lädirt, und sämmtliche 100 Procente beruhen auf dem einen Fall. Es ist dieses ganz richtig und ist die nothwendige Consequenz davon, dass ich in meinen Tafeln keine Schemata sondern die Resultate meiner procentischen Berechnung darstellen wollte, wie ausdrïcklich hervorgehoben wurde. Die in Rede stehende Stelle befindet sich hart neben einzelnen Rindenantheilen, welche in keinem meiner Fälle Sitz einer Läsion waren, wie dieses auf den ersten Blick in die Augen springt, da diese mit Nummern statt mit einem Helligkeitston belegt sind.

1) "Unter anderen in diesem Buche enthaltenen seltsamen Dingen." 
420 Sigm. Exner: Zur Frage nach d. Rindenlocalisation b. Menschen.

Jeder, der weiss worauf die „Methode der procentischen Berechnung" basirt, erkennt, dass ihre Resultate in Fällen, in denen die Berechnung auf wenigen Fällen beruht, werthlos sind. So sage ich pag. 25: „Dass ein Theil der Orbitalwindungen verhältnissmässig dunkel erseheint, muss ich vorläufig für zufällig halten. Es sind nämlich nur zwei, theilweise vier Fälle, in welchen diese Stelle überhaupt Sitz einer Läsion ist." Analoge Aeusserungen, welche zeigen, dass ich die auf wenigen Fällen beruhenden Procentzahlen für werthlos halte, befinden sich auf pag. 39, auf pag. 42 u. a. m. Ich musste die oben genannte Stelle, obwohl sie nur einmal erkrankt war, so schattiren als wäre sie in hundert Procenten der Fälle mit Motilitätsstörung der oberen Extremität verbunden, weil es in der That 100 Procente waren. Eines groben Fehlers würde ich mich aber dann schuldig gemacht haben, wenn ich aus diesen 100 Procenten irgend eine Folgerung gezogen bätte. Dieses behauptet nun Ferrier in der That, befindet sich in dieser Beziehung aber im Irrthum. Ieh habe aus jenen hundert Procenten keinen Schluss gezogen. Es geht das schon daraus hervor, dass ich eben von den 100 Procenten dieser Rindenstelle und von dieser selben Tafel sagte: "Es beruht dies wieder auf einer jener Unregelmässigkeiten, welcher man bei Anwendung meiner Methode gewärtig sein muss, wenn nur wenige Läsionen einer Stelle zur Verfïgung stehen." Ferrier warde, wie es scheint, zu seinem Irrthum durch meine Worte verleitet: "dass übrigens dieser Rindentheil ...". Hierbei bezieht sich "dieser Rindentheil" nicht auf jene Stelle, welche 100 Procent zeigt, sondern auf den kurz vorher genannten Occipitallappen. Es ergiebt sich das nicht nur aus dem Sinn der ganzen Stelle sondern auch aus einem auf pag. 32 meines Buches stehenden Satze, der das Gegentheil von dem aussagt, was Ferrier mich aus jenen 100 Procenten folgern lässt.

Es ist also die Methode der procentischen Berechnung, auf das Gehirn angewendet, sebr wohl brauchbar, obgleich sie hier, sowie in der Nationalökonomie und Statistik, wenn sie nur auf wenigen Fällen basirt, unzuverlässige Resultate giebt.

Ferrier schliesst sein Referat mit den Worten: "Though Prof. Exner advocates the localisation of function in the cerebral hemispheres, the support he gives it is of the most equivocal description" 1). Dieser Bemerkung gegenüber möchte ich hervor-

1) „Obgleich Prof. Exner als Anwalt für die Localisation der Func- 
Karl Lehmann: Die Wirkung hoher Sauerstoffdrücke auf thier. Gebilde. 421

heben, dass ich mich niemals als Anwalt der Localisationslehre gefühlt habe, dass, als ich meine Untersuehungen über diesen Gegenstand begann, es mir ganz gleichgiiltig war, ob die Resultate derselben die eine oder die andere Lehre unterstiitzen werden, und dass es überhaupt ein Lehrsatz der deutschen Naturforschung ist, nicht für eine irgendwie aufgestellte Anschauung Gründe auf Gründe zu häufen, denn da würde der Fleiss, nicht die Wahrheit den Ausschlag geben - sondern eine correcte Fragestellung und zuverlässige Methoden auszusinnen, und die Beantwortung der Frage der Natur selbst zu überlassen. Uebrigens ist die Thatsache von der Rindenlocalisation ganz unabhängig von der Lehre dass „die Centren " nicht ineinanderliegen dürfen, oder dass dieselben eine scharfe Begränzung haben müssen.

(Aus dem physiologischen Laboratorium in Zürich.)

\section{Die Wirkung hoher Sauerstoffdrücke auf thierische Gebilde.}

Von

\section{Karl Iehmann,}

med. pract., Assistent am physiologischen Laboratorium in Zïrich.

In seinem Werke: "La pression barometrique, Paris $1878^{\text {" }}$ hat Paul Bert den schädlichen Einfluss hohen Luftdrucks auf eine giftige Wirkung des comprimirten Sauerstoffs zurückgefihrt. Seine Versuche erstrecken sich über das ganze Thierreich, namentlich sind aber Vögel und Hunde von ihm berücksichtigt worden. Ueberall zeigte sich, dass eine Erhöhung des Sauerstoffpartiar-

tionen in den Gehirnhemisphären auftritt, so ist doch die Unterstützung, welche dieser Lehre durch ihn zu Theil wird, von höchst zweifelhaftem Werthe. 